\title{
Trivium
}

Revue franco-allemande de sciences humaines et sociales - Deutsch-französische Zeitschrift für Geistesund Sozialwissenschaften

$30 \mid 2019$

La constitution au tournant des XXe et XXle siècles

\section{Das Verfassungsrecht: Recht der Verfassung und Verfassung des Rechts}

\section{Louis Favoreu}

Traducteur : Olivier Joop

\section{(2) OpenEdition}

Journals

Édition électronique

URL : http://journals.openedition.org/trivium/6623

DOI : $10.4000 /$ trivium.6623

ISSN : 1963-1820

Éditeur

Les éditions de la Maison des sciences de l'Homme

Référence électronique

Louis Favoreu, "Das Verfassungsrecht: Recht der Verfassung und Verfassung des Rechts », Trivium [Online], 30 | 2019, online erschienen am 18 Dezember 2019, abgerufen am 08 September 2020. URL http://journals.openedition.org/trivium/6623 ; DOI : https://doi.org/10.4000/trivium.6623

Ce document a été généré automatiquement le 8 septembre 2020

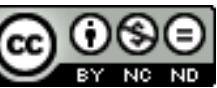

Les contenus des la revue Trivium sont mis à disposition selon les termes de la Licence Creative Commons Attribution - Pas d'Utilisation Commerciale - Pas de Modification 4.0 International. 


\title{
Das Verfassungsrecht: Recht der Verfassung und Verfassung des Rechts
}

\author{
Louis Favoreu
}

Traduction : Olivier Joop

\section{NOTE DE L'ÉDITEUR}

Wir danken den Rechtsnachfolgern von Herrn Louis Favoreu, insbesondere Frau Wanda Mastor, für die freundliche Genehmigung, diesen Artikel in deutscher Übersetzung zu publizieren.

Nous remercions les ayants droit de Monsieur Louis Favoreu, et notamment Mme Wanda Mastor, de nous avoir accordé l'autorisation de traduire ce texte pour le présent numéro.

1 Im Jahr $1980^{1}$ erschien aus dieser Feder eine Abhandlung, in der festgestellt wurde, dass während der vorangegangenen Dekade das »öffentliche Recht eine so tiefgreifende Veränderung erfahren hat, dass man bald von einer >Revolution` wird sprechen können«, und im selben Jahr, anlässlich der ersten Tagung der Association française des Constitutionnalistes (Vereinigung der französischen Staatsrechtslehrer), ${ }^{2}$ haben wir den Wandel des Inhalts und der Stellung des Verfassungsrechts im Verhältnis zu den anderen Rechtsgebieten nachgezeichnet.

2 Nun, zehn Jahre später und vor dem Hintergrund der erheblich gewachsenen Bedeutung der Rechtsprechung des Verfassungsrates, ${ }^{3}$ ist ersichtlich, dass diese Feststellungen nur bekräftigt und weiterentwickelt werden können. Nicht nur wurden die Umwälzungen der Grundgegebenheiten des öffentlichen Rechts und die Wesensänderung des Verfassungsrechts bestätigt, sondern es kann darüber hinaus mit Fug und Recht behauptet werden, dass sämtliche Rechtsbereiche ${ }^{4}$ nunmehr dem Einfluss durch die Verfassung und deren Recht ausgesetzt sind. 
3 Manche Autoren haben eine Rückkehr des Verfassungsrechts alten Stils aus der Vorkriegszeit gewittert oder gar - in einer erstaunlichen Gleichsetzung - ein Zeichen für die Wiederkehr der Rechtsschulen sehen wollen! ${ }^{5}$

4 Eine solche Einstellung hieße jedoch, die grundlegende Entwicklung, die sich seit ungefähr vierzig Jahren im Verfassungsrecht der europäischen Staaten, und darüber hinaus sogar in der Mehrzahl der demokratischen oder zur Demokratie strebenden Staaten, vollzieht, zu verkennen - oder sie völlig unbeachtet zu lassen. Vereinfacht gesagt, hat sich das Wesen der Verfassung gewandelt, ist diese nicht mehr nur eine »Idee«, sondern nun auch eine "Norm«, mit anderen Worten eine als verbindlich anerkannte rechtliche Regelung, »deren Auswirkungen sowohl im Hinblick auf die Bürger als auf die Gerichte spürbar sind $« .^{6}$ Fraglos war dies in Frankreich zur Zeit der III. Republik nicht der Fall, und es ist schwer zu sehen, wie die Verfassungsrechtler jener Zeit die Bestimmungen der Verfassungsgesetze von 1875 auf dieselbe Art hätten betrachten können. Darüber hinaus kann heutzutage die Betrachtungsweise des Verfassungsrechts nicht mehr dieselbe wie damals sein, angesichts der Tatsache, dass die Politikwissenschaft seitdem - unbestrittenerweise - wertvolle Beiträge für die Kenntnis des Verfassungsrechts geleistet hat.

5 Das Phänomen der Verrechtlichung ${ }^{7}$ des Verfassungsrechts steht in engem Zusammenhang mit dem Ausbau der Verfassungsgerichtsbarkeit: Diesbezüglich wird offenbar, wie sehr das Verfassungsrecht in Deutschland, Österreich, Kanada, Italien, Spanien, Griechenland, Portugal und Belgien eine erhebliche Entwicklung erfahren hat (bzw. noch erfährt), wohingegen das britische, das niederländische oder auch das schwedische Verfassungsrecht praktisch unverändert geblieben sind. Wer diese Veränderungen insbesondere durch rechtsvergleichende Untersuchungen wahrgenommen hat, wird daher kaum der Ansicht von Michel Troper beipflichten, ${ }^{8}$ wonach die verfassungsrechtliche Situation die gleiche sei, ob es nun eine Verfassungsgerichtsbarkeit gebe oder nicht. Um die Stichhaltigkeit dieser Aussage in Zweifel $\mathrm{zu}$ ziehen, genügt es, folgende einfache Frage zu stellen: Könnte es ein Verwaltungsrecht ohne Verwaltungsgerichtsbarkeit geben? Sicherlich gilt, wie Georges Vedel ${ }^{9}$ treffenderweise aufgezeigt hat, dass die Lage, in der sich der Verfassungsrichter gegenüber der Verfassung befindet, nicht völlig dieselbe ist wie diejenige des Verwaltungsrichters gegenüber dem Verwaltungsrecht, jedoch ist schwer vorstellbar, wie ohne den einen wie den anderen die Verwaltung und der Gesetzgeber zur Einhaltung des Rechts hätten verpflichtet werden können.

6 Sicherlich ist die Entwicklung - die nicht älter als dreißig Jahre ist - noch nicht abgeschlossen, doch ist es durchaus möglich, zu Beginn dieser 1990er Jahre Bilanz zu ziehen oder zumindest eine Bestandsaufnahme zu erstellen. Dazu wird im Folgenden das Verfassungsrecht zwei Betrachtungsweisen unterzogen, zunächst als »Recht der Verfassung«, sodann als "Verfassung des Rechts«.

\section{Das Verfassungsrecht, Recht der Verfassung}

71949 schrieb Georges Vedel in seinem Lehrbuch Verfassungsrecht (S. 5):

"[I]n den meisten Ländern und hinsichtlich der meisten Bereiche findet sich das Recht, das die Staatsgewalt regelt, in einer geschriebenen Verfassung niedergelegt. Es ist dieses umfängliche und sehr häufige Zusammentreffen, das zur Einführung 
und Verwendung des Begriffs des Verfassungsrechts geführt hat, obgleich das

Verfassungsrecht etwas anderes als das \Recht der Verfassung « ist «.

$\mathrm{Zu}$ Beginn der IV. Republik ist das »Recht der Verfassung « nicht so umfassend wie das Verfassungsrecht: Vierzig Jahre später kann jedoch umgekehrt behauptet werden, dass das Verfassungsrecht - zumindest so, wie es üblicherweise verstanden wird - nunmehr $\mathrm{zu}$ eng ist, um das gesamte »Recht der Verfassung« zu umfassen. Der Gegenstand des Verfassungsrechts hat sich verändert.

Der Terminus »Recht der Verfassung« wird hier ebenfalls zur Definition des Verfassungsrechts zu Beginn dieser 1990er Jahre dienen.

\section{A. Der Gegenstand des Verfassungsrechts}

9 In seiner »Vorlesung zum Vergleichenden Verfassungsrecht « von 1950/51 hat Charles Eisenmann richtigerweise - namentlich in Bezug auf das Verfassungsrecht hervorgehoben, dass Recht und Rechtswissenschaft nicht miteinander verwechselt werden dürfen ${ }^{10}$ :

»Der Begriff >Recht` für und in sich selbst betrachtet, und mehr noch in zusammengesetzten Begriffen wie Zivilrecht, Verfassungsrecht oder Verwaltungsrecht, wird fortwährend in einem zweifachen Bedeutungssinn verwendet, wobei häufig von der einen zur anderen dieser Bedeutungen gewechselt wird. Manchmal bezieht sich der Begriff auf einen Korpus von Regeln, auf die Regeln in ihrer Gesamtheit oder auf bestimmte Rechtsregeln. Dann wieder bezeichnet er die Disziplin(en), deren Gegenstand diese Regeln sind; in diesem Fall bezeichnet er die Untersuchung oder die Wissenschaft von diesem Korpus von Regeln. Dieser doppelte Wortsinn findet sich insbesondere bezüglich der Bezeichnung >Verfassungsrecht $\iota . \ll$

\section{Von einem einzigen zu drei Gegenständen}

10 Fürs Erste kann die Idee des Zusammenhangs zwischen dem »Korpus von Regeln« und der »Disziplin, deren Gegenstand diese Regeln sind«, beibehalten werden. Von diesem Blickwinkel aus betrachtet ist unbestreitbar, dass der Gegenstand des Verfassungsrechts (zumindest in Frankreich) zum Ende der 1940er Jahre ein ganz anderer war als zu Beginn der 1990er Jahre: Es kann sogar behauptet werden, dass dieser Gegenstand nunmehr aus drei Teilen besteht, da im Vergleich zum Verfassungsrecht der IV. Republik, das sich hauptsächlich mit der Organisation der Staatsorgane befasste, im Verfassungsrecht der V. Republik - diesbezüglich auf einer Linie mit dem heute allgemein üblichen Verfassungsrecht - zur Staatsorganisation noch der Bereich des Normengefüges und der Bereich des Grundrechtsschutzes hinzugetreten sind. Im Interesse besserer Übersichtlichkeit ${ }^{11}$ soll hier im Folgenden daher, der Dreigliedrigkeit des modernen Verfassungsrechts - Organe, Normen, Grundrechte - folgend, zwischen dem Staatsorganisationsrecht, dem normativen Verfassungsrecht und dem materiellen Verfassungsrecht unterschieden werden.

\section{a) Die Institutionen: Das Staatsorganisationsrecht}

11 Hierbei handelt es sich um den klassischen Gegenstand des französischen Verfassungsrechts seit dem letzten Krieg: die Darstellung der politischen Institutionen nach vier Themengebieten, nämlich der allgemeinen Staatslehre, ${ }^{12}$ den ausländischen politischen Institutionen, der Geschichte der französischen politischen Institutionen 
sowie den politischen Institutionen der V.Republik. Die Vorlesung zum Verfassungsrecht ist üblicherweise wesentlich eine Vorlesung zum Staatsrecht, obgleich die offizielle Bezeichnung dieser Lehrveranstaltung im Rahmen des ersten Studienjahres der Rechtswissenschaften folgendermaßen lautet: »Verfassungsrecht und politische Institutionen«.

Rechtsfragen werden im Allgemeinen gegen Ende der Vorlesung zur V. Republik erörtert, seien es Fragen in Bezug auf das Normengefüge (Theorie der Rechtsquellen, Stufenbau der Rechtsordnung) oder auch Rechtsfragen $\mathrm{zu}$ den Institutionen selbst. Zwar werden üblicherweise die Bestimmungen zur Einteilung der Wahlkreise, zur Parteien- und Wahlkampffinanzierung, zum aktiven und passiven Wahlrecht usw. thematisiert, allerdings wird auf die rechtlichen Fragestellungen $\mathrm{zu}$ diesen Themen eher weniger eingegangen.

Darüber hinaus beschränkt sich das Studium der Institutionen auf die politischen Institutionen. Dabei erstreckt sich das Verfassungsrecht, so wie es heutzutage in der Mehrzahl der Länder verstanden wird, auch auf die Einrichtungen der Verwaltung (insbesondere auf lokaler Ebene) und die Organe der rechtsprechenden Gewalt. Sicherlich hängt - insbesondere in Bezug auf jene - viel von der Staatsform ab (Bundesstaat, quasi-bundesstaatliche Ordnung oder Ordnung mit autonomen Gebietskörperschaften, Einheitsstaat); doch selbst in einem Einheitsstaat wie Frankreich ist es heutzutage nicht mehr möglich, die Frage der verfassungsrechtlichen Grundlagen der Verwaltungseinrichtungen sowie des Gerichtswesens unberücksichtigt zu lassen.

14 Es stimmt zwar, dass in diesem Bereich die Tradition in Frankreich gegen eine solche Einbeziehung der Verwaltungsbehörden und der Gerichte in das Verfassungsrecht spricht. Diese Haltung wird damit begründet, dass die Verfassung nur Bestimmungen über die politischen Institutionen enthalten könne. ${ }^{13}$ Jedoch ist eine solche Auffassung in der Rechtsvergleichung und selbst in der gegenwärtigen französischen Verfassungslehre inzwischen völlig überholt. Wer könnte in der Tat heute, nach den Entscheidungen des Verfassungsrates vom 25. Februar $1982^{14}$, vom 22. Juli $1980^{15}$ oder auch vom 23. Januar $1987^{16}$ - um nur die bedeutendsten zu nennen -, noch ernsthaft behaupten, die verfassungsmäßigen Grundlagen der Gerichtsorganisation seien kein Gegenstand einer wissenschaftlichen Befassung mit dem Verfassungsrecht?

Zusammenfassend lässt sich festhalten, dass $\mathrm{zu}$ den Themen des Staatsorganisationsrechts die politischen Institutionen und die damit im Zusammenhang stehenden Rechtsfragen ebenso gehören wie die Fragen bezüglich der verfassungsmäßigen Grundlagen der Verwaltungseinrichtungen und des Gerichtswesens.

\section{b) Die Normen: das Normengefüge im Verfassungsrecht}

Das normative Verfassungsrecht bzw. das grundlegende Verfassungsrecht sollte eigentlich unter den Regelungsgehalten des Verfassungsrechts an erster Stelle genannt werden, doch ist es - in Frankreich - so wenig üblich, es dem Verfassungsrecht in all seinen Dimensionen zuzuordnen, dass es hier an zweiter Stelle behandelt werden soll.

Es umfasst, was manchmal auch als das System der Rechtsquellen ${ }^{17}$ bezeichnet und hier besser unter dem Begriff des Normensystems behandelt werden wird. Im französischen Recht setzt sich nach und nach die Erkenntnis durch, dass das System der 
Rechtsquellen von der Verfassung abhängt, eine Tatsache, die in der Rechtsvergleichung bereits seit langem anerkannt ist. ${ }^{18}$

Das Normensystem bildet ein komplexes Netzwerk, in der V. Republik bemerkenswert strukturiert durch die Verbindung der Artikel 2, 11, 16, 34, 37, 38, 41, 45, 46, 53, 54, 55, 72, 73, 74, 89 und 92 der Verfassung: Die Art und Weise, wie die nationalen und die lokalen Normen, die nationalen und die internationalen Normen sowie die unterschiedlichen Kategorien nationaler Normen untereinander angeordnet sind, einander ergänzen und ineinander greifen, erinnert an ein Uhrwerk, das vom Verfassungsrichter justiert wird. Wie wir im Rahmen der Einführung zum Symposium über die »Abfassung der Verfassung« hervorgehoben haben, liegt hier ein ausgefeilter Mechanismus vor, dessen Einzelteile von den "rechtsgelehrten Richtern « des Staatsrats, die an der Ausarbeitung der Verfassung beteiligt waren, mit besonderer Sorgfalt »bearbeitet« worden sind und der von den Richtern des Verfassungsrates in die Praxis umgesetzt wird. ${ }^{19}$ Die Möglichkeiten und die Komplexität des französischen Normengefüges offenbaren sich nur nach und nach, im Zuge der sich entwickelnden Verfassungsrechtsprechung. ${ }^{20}$

Im Rechtsvergleich finden sich jedoch noch komplexere Systeme von Rechtsquellen, namentlich in Bundesstaaten oder Staaten mit bundesstaatsähnlicher Organisationsform.

Zusammenfassend lassen sich mindestens drei Situationen unterscheiden, je nachdem, $\mathrm{ob}$ es eine Kategorie von Rechtsquellen (nationale Rechtsquellen, wie z. B. in Japan), zwei Kategorien von Rechtsquellen (nationale und internationale, wie z.B. in Frankreich oder den Niederlanden, oder auch nationale und lokale wie in den Vereinigten Staaten) oder drei Kategorien von Rechtsquellen (internationale, nationale und lokale, wie z. B. in der Bundesrepublik Deutschland, in Italien oder in Spanien) gibt. Wie viele und welche Rechtsquellen es gibt, hängt von der Verfassung und damit vom Verfassungsrecht ab. Auch ist es entbehrlich, die Bedeutung des Systems der Rechtsquellen in den verschiedenen Ländern hervorzuheben: es ist mindestens genauso bedeutsam wie das Staatsorganisationsrecht.

\section{c) Die Grundrechte: das materielle Verfassungsrecht}

Unter dieser Bezeichnung soll hier das die Grundfreiheiten schützende Verfassungsrecht verstanden werden, das wir bei anderer Gelegenheit auch als die Beziehungen zwischen Staat und Bürger regelndes Verfassungsrecht bezeichnet haben. ${ }^{21}$ In Frankreich ist es nicht üblich, die Grundfreiheiten im Rahmen des Verfassungsrechts $\mathrm{zu}$ thematisieren. Im Rechtsvergleich hingegen ist dies eher die Ausnahme als die Regel, denn in der Mehrzahl der modernen westlichen Rechtssysteme stellen die Beachtung und der Schutz der Grundrechte einen der wichtigsten Zweige des Verfassungsrechts dar.

Diese Aspekte gehören zu den Anforderungen, die ein modernes Verfassungsrecht erfüllen muss. Sicherlich könnte der Gedanke an eine Wiederkehr der Rechtslehren Duguits und Haurious aufkommen, die in den Menschenrechtserklärungen Verfassungsrecht oder gar Über-Verfassungsrecht sahen; doch wie Eisenmann bereits 1928 aufzeigen konnte, entsprangen derartige Theorien eher der Naturrechtslehre als dem Rechtspositivismus und hatten eine stärker politische denn rechtliche Stoßrichtung. ${ }^{22}$ Heutzutage hingegen geht die Eingliederung der Grundrechte und - 
freiheiten auf eine bewusste Entscheidung des Verfassungsgebers zurück, der diesen ausdrückliche Bestimmungen im Verfassungstext selbst widmet, ebenso wie den Staatsinstitutionen und dem Normengefüge. ${ }^{23}$

Darin kommt ein Wandel zum Ausdruck in Bezug auf das Verständnis der Befugnisse der Regierenden, denn dem Einzelnen in der Verfassung verankerte Grundrechte und freiheiten zuzuerkennen bedeutet zwangsläufig eine erhebliche Einschränkung der Macht der Regierenden. Diese haben nicht nur Form- und Verfahrensvorschriften zu beachten, sondern auch materielle Rechtsvorschriften. Der Einzelne vertraut der Mehrheit nicht mehr, selbst wenn diese aus einem vollständig demokratischen Verfahren hervorgegangen ist: Er will, dass die Entscheidungen der Mehrheit überprüft werden können, damit sichergestellt ist, dass seine Grundrechte nicht verletzt werden.

\section{Der Wesenswandel des Verfassungsrechts}

Zusammenfassend lässt sich feststellen, dass sich der Gegenstand des Verfassungsrechts der III. und der IV. Republik auf »die Institutionen, mittels deren die Staatsgewalt begründet, ausgeübt und übertragen wurde « ${ }^{24}$ beschränkte. In gewisser Hinsicht ging es lediglich darum $\mathrm{zu}$ überprüfen, dass die Staatsorgane in demokratischer Weise eingerichtet worden waren und die Wahl der Volksvertreter so unverfälscht wie möglich durchgeführt worden war. ${ }^{25}$ So erklärt sich die Bedeutung, die Fragen der Durchführung von Wahlen, der Parteien und der verschiedenen möglichen politischen Akteure, sowie Fragen institutioneller Mechanismen wie die zur Sicherung der Verantwortlichkeit der Regierung gegenüber dem Parlament, die Auflösung der Volksvertretung oder, allgemeiner, Fragen der Beziehungen zwischen den Staatsgewalten beigemessen wird.

Diese Feststellung gilt für den Inhalt und die Struktur der Verfassungen von 1875 und 1946: Diese Verfassungen hatten überwiegend die Organisation des Staates zum Gegenstand und sollten dafür sorgen, dass sich der allgemeine Wille bestmöglich durch vorschriftsmäßig gewählte Abgeordnete ausdrücken konnte. Darin folgten sie dem britischen Demokratieverständnis.

Diese Auffassung gründet auf einer »optimistischen« Grundannahme, nämlich dass der von der Mehrheit ausgedrückte Wille dem allgemeinen Willen entspricht und Minderheiten von der Mehrheit weder unterdrückt noch benachteiligt werden. Die Briten heben auch hervor, dass die politische Kultur der Bevölkerung dazu beiträgt, dass der von der Mehrheit formulierte Wille "gesittet« ausfällt und Beschränkungen unterliegt. Eine pessimistische Sicht ist freilich ebenso denkbar, die in der demokratischen Staatsform einen Nutzen nur für einen Teil der Bevölkerung sieht. So kam es in Kanada anlässlich der Ausarbeitung einer eigenen Verfassung als Abkehr vom reinen britischen Verfassungsmodell zur Aufnahme einer Grundrechtserklärung in den Verfassungstext, die auf eine Initiative des Premierministers P.E. Trudeau zurückging, von einigen seiner politischen Gegner allerdings im Namen der reinen Demokratielehre britischen Typs bekämpft worden war. W. F. Murphy hat dazu sehr klar ausgeführt ${ }^{26}$ :

»Im Grunde genommen würden die Verfassungsrechtler annehmen, dass beide Vorgehensweisen richtig wären; sicherlich wäre jede von ihnen geeignet, um den Schutz der Rechte der weißen, englischsprachigen, im Land geborenen Angehörigen der Mittelklasse zu gewährleisten. Aber in England würden mich Iren, Indianer und Schwarze nicht ernstnehmen, wenn ich behauptete, ihre Rechte seien 
ebenso gut geschützt wie diejenigen der französisch-, japanisch-, inuit- oder indianisch-stämmigen Kanadier.« Verfassungsrecht zu bringen. Dazu sollen die folgenden drei Thesen näher entwickelt werden, nämlich dass es sich beim Verfassungsrecht um Recht handelt (1.), und zwar das Recht der Verfassung (2.), ein Recht, das durch Gerichte durchgesetzt werden kann (3.).

\section{Verfassungsrecht als Recht: die Abkehr von der auf die Politikwissenschaft zentrierten Sichtweise}


täten, dann nur aus einer Art Standesdünkel heraus und um »das Feld zu besetzen« und andere Fachleute (namentlich die Politikwissenschaftler) vom Diskurs auszuschließen, wodurch sie diesen gewissermaßen zu ihren Gunsten zu monopolisieren suchten.

Hier ist zunächst festzustellen, dass sich die Politikwissenschaftler - oder zumindest einige unter ihnen - in der Defensive befinden (was ihnen seit langem nicht widerfahren war) und sich im Übrigen allgemein einer gleichsam militärisch inspirierten Rhetorik bedienen, wenn sie von »intellektuellen und universitären Strategien $\aleph^{29}$ oder von $» P u t s c h \ll$ usw. sprechen.

Ernsthafter, und auf der Sachebene, drückt diese Kritik eine Sichtweise aus, die man als politikzentriert bezeichnen könnte und nach deren Verständnis Phänomene der Verfassung und der Politik nur durch die Politikwissenschaft erfasst und betrachtet werden sollten. Doch selbst wenn unterstellt würde, dass der Ausschließlichkeitsanspruch der politikwissenschaftlichen Herangehensweise begründet wäre, müsste dennoch festgehalten werden, dass diese Herangehensweise nicht zielführend wäre, da von einem bestimmten Kontext abhängig und darüber hinaus auch veraltet.

35 Zum einen ist eine solche Herangehensweise sehr eng mit Frankreich und dessen Besonderheiten verknüpft: Die Politikwissenschaftler stützen ihre Denkmodelle hauptsächlich auf die (klassische) französische Auffassung des Verfassungsrechts und nicht auf die heute nicht nur in den Vereinigten Staaten, sondern auch in den meisten westeuropäischen Staaten mit einer Verfassungsgerichtsbarkeit vorherrschende Sicht. Diesbezüglich ist anzumerken, dass den französischen Verfassungsrechtlern $\mathrm{zu}$ unterstellen, sie verwendeten besondere Begrifflichkeiten und eine besondere - gar codierte -Sprache, um eine "Abschottung ihrer wissenschaftlichen Disziplin" herbeizuführen, darauf hinausliefe, sie einer über mindestens vierzig Jahre hin gesponnenen und groß angelegten Verschwörung zu bezichtigen, die beabsichtigte, die österreichischen, deutschen, italienischen, schweizerischen und natürlich auch die amerikanischen, sowie heutzutage die spanischen, portugiesischen, griechischen, belgischen usw. Verfassungsrechtler dazu zu bringen, dasselbe Vokabular wie sie zu verwenden (und dies sogar viele Jahre früher als sie selbst).

Doch selbst aus rein innerfranzösischer Perspektive ist diese Herangehensweise veraltet und bereits dadurch auch ungeeignet und erfasst nur einen Teil des Problems. Zum einen nämlich fokussieren sich die Politikwissenschaftler auf einen Begriff des Verfassungsrechts, der im Wesentlichen auf der Exegese des Verfassungstextes basiert, und sehen in der gegenwärtigen Neubelebung des Verfassungsrechts eine Rückkehr zur Vorgehensweise aus der Vorkriegszeit. Allerdings berücksichtigt eine derartige Sicht nicht die Tatsache, dass das heutige Verfassungsrecht nicht auf einer Exegese des Verfassungstextes durch die Rechtslehre beruht, sondern auf einer reichhaltigen Auslegung durch die Rechtsprechung der (Verfassungs)Gerichte, die es so bislang nicht $\mathrm{gab}^{30}$ und die als solche auch nicht Gegenstand politikwissenschaftlicher Betrachtungen gewesen ist. ${ }^{31}$ Sodann ist anzumerken, dass die Politikwissenschaftler dem Verfassungsrecht nur in dem Maß Beachtung schenken, wie es die Institutionen des Staates regelt, was heißt, dass dabei nur einer der drei Gegenstände des modernen Verfassungsrechts betrachtet wird. Da sich die gesamte Aufmerksamkeit der Politikwissenschaftler und der "Institutionalisten « auf das Wahlsystem, die politischen Parteien, das Parlament, die Regierung und die Beziehungen zwischen diesen Staatsorganen konzentriert, bilden das normative Verfassungsrecht und das materielle 
Verfassungsrecht einen blinden Fleck in ihren wissenschaftlichen Untersuchungen. Selbst wenn es also den Politikwissenschaftlern gelänge, ohne Berücksichtigung der Verfassungsrechtsprechung Schlüsse aus ihrer Analyse der Institutionen zu ziehen, wären diese Schlüsse doch nur unvollständig und in Bezug auf die Gesamtheit dessen, was heutzutage das Verfassungsrecht ausmacht, nicht stichhaltig.

b) Da für sie das Verfassungsrecht als ein Bereich des Rechts ein Forschungsgegenstand ist, müssen die Verfassungsrechtler auch auf die Terminologie des Rechts zurückgreifen, die im Übrigen nicht weniger unzugänglich ist als diejenige der Politikwissenschaftler. Darüber hinaus kann die Notwendigkeit auftreten, neue Vorstellungen des modernen Verfassungsrechts auch mit neuen Begrifflichkeiten $\mathrm{zu}$ erfassen. ${ }^{32}$

So dürfen beispielsweise die Begriffe Oberster Gerichtshof und Verfassungsgericht nicht verwechselt werden ${ }^{33}$ : Es handelt sich um zwei Arten der Ausübung von Verfassungsgerichtsbarkeit (letztere Bezeichnung dient hierbei als Oberbegriff), die zu zwei unterschiedlichen Modellen gehören. Es käme auch niemandem in den Sinn, beispielsweise von einem "parlamentarischen Präsidialsystem « zu sprechen; gleichfalls ist es unmöglich, von einem "Obersten Verfassungsgerichtshof" $\mathrm{zu}$ sprechen. Parlamentarische Demokratie und präsidiale Demokratie sind zwei verschiedene Formen eines freiheitlichen Regierungssystems, aber dies darf nicht dazu führen, sie durcheinanderzubringen; Gleiches gilt, in Bezug auf die Verfassungsgerichtsbarkeit, für Oberste Gerichte und Verfassungsgerichte.

\section{Das Verfassungsrecht als Recht der Verfassung: die Abkehr von der auf die Verwaltung zentrierten Sichtweise}

39 a) Lange Zeit war das Verwaltungsrecht die dominierende Disziplin im öffentlichen Recht, und es ist nur $\mathrm{zu}$ verständlich, dass sich seine Vorstellungen und Begriffsbestimmungen auch durch die Rechtsprechung des Staatsrates durchgesetzt haben. Doch ab dem Moment, wo sich eine im Wesentlichen auf ein Verfassungsrecht, wie es im Rechtsvergleich bereits seit längerem vorherrscht, stützende Verfassungsrechtsprechung etabliert, verändert sich auch die Gemengelage: die Vorstellungen und Begriffe sind nicht dieselben, denn das Verfassungsrecht ist nicht das Verwaltungsrecht.

Ein Beispiel soll dies verdeutlichen: Der im Rechtsvergleich häufig verwendete Begriff der "Grundfreiheiten« oder auch der »Grundrechte« ist nicht deckungsgleich mit dem Begriff der »bürgerlichen Freiheiten" (libertés publiques); Letzterer verweist im Wesentlichen auf Freiheitsrechte, die gesetzlich (und von den ordentlichen Gerichten oder den Verwaltungsgerichten) gegen Eingriffe der Exekutive geschützt werden, während die beiden erstgenannten Begriffe Freiheiten bezeichnen, die gemäß der Verfassung oder völkerrechtlichen Übereinkommen von einem Verfassungsgericht (bzw. einem internationalen Gericht) gegen Eingriffe durch die Exekutive und die Legislative geschützt werden.

41 Desgleichen ist es nicht möglich, auf der Grundlage der im Verwaltungsrecht verwendeten Begriffe und Vorstellungen die Verfassungsgerichtsbarkeit zu definieren: deren Wesensmerkmale müssen anhand der Theorie von der Verfassungsgerichtsbarkeit abgeleitet werden, wie sie sich auf der Grundlage des Rechtsvergleichs ergibt. Niemand würde eine politische Ordnung als 
"parlamentarisches" oder "präsidiales" System qualifizieren, ohne sich auf die allgemeinen und in der Rechtsvergleichung gebräuchlichen Kriterien zu beziehen, die ein parlamentarisches bzw. ein präsidiales Regierungssystem ausmachen. Daher ist auf eine sogenannte "auf die Verwaltung zentrierte Sichtweise ${ }^{34} \mathrm{zu}$ verzichten: Die Begriffe und Vorgehensweisen im Verfassungsprozessrecht sind sicherlich in hohem Maße denjenigen des Verwaltungsprozessrechts entlehnt, doch kann keine vollständige oder zwangsläufige Übereinstimmung zwischen diesen beiden Prozessrechten abgeleitet werden. Tatsächlich ist das Verwaltungsprozessrecht vom innerstaatlichen Recht geprägt und spezifisch für die französische Verwaltungsgerichtsbarkeit, während das Verfassungsprozessrecht vom Rechtsvergleich inspiriert ist und nur durch dieses verständlich wird.

b) Dies soll nicht bedeuten, dass das Verwaltungsrecht die Verfassungsrechtsprechung, und damit im Weiteren auch das Verfassungsrecht selbst, nicht »befruchten« würde.

In der Tat ist es so, dass die französische Verfassungsgerichtsbarkeit - in diesem Punkt einer Erscheinung aus dem italienischen Recht und in diesem als "Lehre vom lebendigen Recht $\aleph^{35}$ folgend - im Recht der Verfassung zweifellos auch Rechtsfiguren und Denkmuster einbezieht, die von der Verwaltungsgerichtsbarkeit oder von der ordentlichen Gerichtsbarkeit geschaffen wurden..$^{36}$ So kann beispielsweise davon gesprochen werden, dass immer häufiger eine »Verfestigung" der von der Verwaltungsgerichtsbarkeit geschaffenen Lösungsansätze durch die Verfassungsgerichtsbarkeit erfolgt. ${ }^{37}$ Auf diese Weise werden die Rechtsfiguren und Vorgehensweisen aus der Verwaltungsrechtsprechung dem Verfassungsrecht einverleibt. Jedoch darf dabei nicht übersehen werden, dass, einmal einverleibt, diese Rechtsfiguren und Vorgehensweisen fortan verfassungsrechtlicher Art sind, und es damit Aufgabe der Verfassungsgerichtsbarkeit ist - und nicht mehr der ordentlichen Gerichtsbarkeit bzw. der Verwaltungsgerichtsbarkeit -, sie weiterzuentwickeln. Eine tiefergehende Analyse zur Feststellung der genauen Tragweite dieser Verfestigung der von der Verwaltungsgerichtsbarkeit aufgestellten Lösungsansätze durch die Verfassungsgerichtsbarkeit wäre sicherlich lohnenswert.

\section{Das Verfassungsrecht als Recht, das durch ein Verfassungsgericht durchgesetzt werden kann: Ablehnung der auf das amerikanische System zentrierten Sichtweise}

a) Das Verfassungsrecht wird mehr und mehr zu einem Recht, das von Gerichten durchgesetzt werden kann. Sicherlich haben auch Großbritannien oder die Niederlande ein Verfassungsrecht, obwohl es in diesen Ländern keine Verfassungsgerichtsbarkeit gibt, die über die Einhaltung der Verfassung wacht; gleichfalls gab es im Frankreich der III. und der IV. Republik auch ohne Verfassungsgerichtsbarkeit ein Verfassungsrecht. Doch das moderne Verfassungsrecht ist heutzutage als Recht schwer vorstellbar ohne eine Gerichtsbarkeit, die dieses Recht durchsetzen kann: Das deutsche, amerikanische, österreichische, kanadische, das spanische, italienische, griechische, portugiesische, das schweizerische usw. Verfassungsrecht basieren gegenwärtig in wesentlichem Maße auf der Verfassungsrechtsprechung; Gleiches wird bald auch in Bezug auf das belgische Verfassungsrecht gelten, da die Zuständigkeiten des Schiedshofes vor kurzem erweitert wurden. Auch in Frankreich ist eine derartige Entwicklung feststellbar: der Verfassungsrichter hat durch seine Tätigkeit, wie in den anderen Ländern mit einer Verfassungsgerichtsbarkeit, das Verfassungsrecht verändert. 
b) Anwendung und Durchsetzung des Verfassungsrechts erfolgen selbstverständlich durch ein Verfassungsgericht, und nicht durch die ordentliche Gerichtsbarkeit. Diese kann sicherlich zur Ausstrahlung des angewendeten Verfassungsrechts beitragen, aber es kann kein im modernen Sinne verstandenes Verfassungsrecht geben ohne Verfassungsgerichtsbarkeit. Unter dem Begriff der Verfassungsgerichtsbarkeit ist in der Rechtsvergleichung jede Form von Gericht zu verstehen, dessen Bestehen, Zusammensetzung und Zuständigkeiten von der Verfassung selbst vorgesehen und gewährleistet werden und dessen Entscheidungen ausschließlich von der verfassungsgebenden Gewalt umgestoßen werden dürfen. So ist beispielsweise gemäß der Rechtsprechung des Verfassungsrates der Bestand der Verwaltungsgerichtsbarkeit durch die Verfassung gesichert, doch bedeutet dies nicht, dass es dem Gesetzgeber durch die Verabschiedung eines ordentlichen Gesetzes nicht freistünde, die Zusammensetzung und das Verfahren für die Auswahl der Richter des Staatsrates oder der Verwaltungsgerichte $\mathrm{zu}$ ändern, die Zuständigkeiten dieser Gerichte $\mathrm{zu}$ modifizieren oder auch eine missliebige Rechtsprechung zu überwinden.

c) In dem Maße, in dem das Verfassungsrecht zu einem von der Rechtsprechung geprägten Recht wird, liegt die Versuchung nahe, den Blick gen Amerika zu richten und die in den Vereinigten Staaten weit verbreiteten Theorien ${ }^{38}$ in Bezug auf die gerichtliche Auslegung der Verfassung zu übernehmen.

Dort kreisen die verfassungsrechtlichen Fragestellungen um die gerichtliche Auslegung der Verfassung, während dieses Thema im Verfassungsrecht in Europa bis vor kurzem nur ein sehr begrenztes oder gar kein Interesse genoss. Hier gilt es, nicht von einem Extrem ins andere zu fallen und davon auszugehen, alles hänge nur noch von einer "zeitgerechten Auslegung" der Verfassung ab. Sicherlich ist der Gewinn durch eine solche Vorgehensweise nicht zu unterschätzen, und die Arbeiten Michel Tropers sind in diesem Zusammenhang von großem Wert. ${ }^{39}$ Die diesbezügliche Debatte in der Rechtslehre dreht sich allerdings nicht um die Frage der Sachdienlichkeit einer solchen Sichtweise, sondern um diejenige der Stellung, die sie im Rahmen verfassungsrechtlicher Fragen einnehmen soll. Hier wird die Ansicht vertreten, dass sie nicht den gesamten Diskurs beherrschen soll und damit die auf das amerikanische System zentrierte Sichtweise ebenso wie die auf die Politikwissenschaft und auf die Verwaltung zentrierte Sichtweise abzulehnen ist, und zwar aus mehreren Gründen.

Zunächst ist festzuhalten, dass die staatsorganisatorischen und rechtlichen Rahmenbedingungen in Europa und in den Vereinigten Staaten nicht die gleichen sind. Die amerikanische Verfassung ist zweihundert Jahre alt und war bereits Gegenstand unzähliger gerichtlicher Entscheidungen, die sie ausgelegt haben, wohingegen die Verfassungen der europäischen Staaten deutlich jünger und Ausdruck des Willens des jeweiligen Verfassungsgebers sind, eine bestimmte rechtliche und politische Ordnung zu schaffen; es ist schwierig, diese Tatsache im Sinne einer zeitgerechten Auslegung der Verfassung, die demjenigen, der diese Auslegung vornimmt, insbesondere den Gerichten, praktisch jedes Ermessen einräumt, nicht zu beachten oder zu übergehen. Des Weiteren besteht die Problematik in den Vereinigten Staaten wesentlich darin, die Art und Weise zu bestimmen, wie die Grundrechte zu berücksichtigen sind und wie deren Beachtung dem Gesetzgeber auferlegt werden kann: Ob nun im Rahmen der Debatte um die sogenannten "neutralen Prinzipien« oder derjenigen zwischen "Interpretivisten" und "Nicht-Interpretivisten«, oder auch bei Fragen, wie sie bereits im Titel eines Werkes des herausragenden Vertreters der amerikanischen 
Rechtsliteratur (R. Dworkin, Bürgerrechte ernstgenommen, dt. 1990) angedeutet werden, stets geht es um die Definition der Grundrechte und die Frage, ob der Richter bei der Bestimmung von deren genauer Bedeutung der Verfassung treu geblieben ist oder nicht. Dieser Ansatz setzt den Schwerpunkt fast ausschließlich auf einen einzigen der Gegenstände des Verfassungsrechts und vernachlässigt die anderen: Er ist deshalb genauso unbefriedigend wie der Ansatz, der den Fokus auf das Staatsorganisationsrecht legt. Schließlich besteht in den Vereinigten Staaten eine derartige Verflechtung zwischen dem Verfassungsrecht und den anderen Rechtsbereichen, dass der Diskurs über den Bedeutungsgehalt der Grundrechte auf eine philosophische Debatte über die Bedeutung des Rechts hinausläuft. In Europa hingegen ist eine derartige Osmose von Verfassungsrecht und übrigem Recht noch in weiter Ferne: Daher können die aus dem amerikanischen Beispiel gezogenen Schlüsse keinen universalen Geltungsanspruch haben und sind nicht ohne weiteres übertragbar.

Richtig ist allerdings ebenfalls, dass das Verfassungsrecht sich zunehmend als Grundlage auch der übrigen Rechtsgebiete etabliert und somit als »Verfassung des Rechts« gelten kann.

\section{Das Verfassungsrecht, Verfassung des Rechts}

Die nun folgenden Ausführungen sollen einen weniger breiten Raum einnehmen, denn es können lediglich die Grundlinien der gegenwärtig erfolgenden Entwicklung skizziert werden. Das Thema soll aber im Weiteren immer wieder Gegenstand von diesbezüglichen Beiträgen in den Chroniken dieser Zeitschrift [Revue française de Droit constitutionnel] sein.

51 Mit fortschreitender Anwendung und Auslegung der Verfassung durch den Verfassungsrichter sowie mit der Umsetzung der derart ausgelegten Verfassungsnormen durch die politischen Organe und die Verwaltungsbehörden findet eine allmähliche verfassungsrechtliche Durchdringung der verschiedenen Rechtsgebiete statt, in deren Folge die Unterscheidung zwischen öffentlichem Recht und Privatrecht an Trennschärfe verliert.

\section{A. Die fortschreitende verfassungsrechtliche Durchdringung der verschiedenen Rechtsgebiete}

1. Was noch $1980^{40}$ sehr skeptisch als einsamer Ruf in der Wüste aufgenommen worden war, trifft zehn Jahre später auf einen breiten Konsens unter Straf-, Verwaltungs-, Handels- wie Arbeitsrechtlern.

Diesbezüglich besonders vielsagend ist der einleitende Hinweis von einem der bedeutendsten Spezialisten für Arbeitsrecht, Professor G. Lyon Caen, zu seiner kürzlich erschienenen Rechtsprechungschronik zum Thema "Die Rechtsprechung des Verfassungsrates mit Bezug zum Arbeitsrecht« (Revue Dalloz, 1989, S. 192, Fn. 1):

»Diese Chronik wird manchen Fachleuten für Verfassungsrecht sehr oberflächlich erscheinen. ${ }^{41}$ Ihr Zweck ist es, den Spezialisten für Arbeitsrecht die verfassungsrechtlichen Grundlagen ihrer Disziplin näherzubringen. Dies ist umso unverzichtbarer, als die Arbeitsgerichte (ebenso wie die übrigen Gerichte), in den Begründungen ihrer Beschlüsse immer stärker auf verfassungsrechtliche Grundsätze Bezug nehmen. Gleichzeitig erklärt sich die grundlegende 
Widersprüchlichkeit innerhalb des Arbeitsrechts durch die Dualität von dessen verfassungsrechtlichen Quellen.«

Im gleichen Sinne kann auch hervorgehoben werden, was der Redakteur der Revue des sociétés in Bezug auf Entscheidungen des Verfassungsrates aus dem Jahr 1986 geschrieben hat, nämlich dass sie »die immer stärkere Rolle belegen«, die dem Verfassungsrat im Bereich des Handelsrechts zukommt, wobei doch gerade dieses Rechtsgebiet »eigentlich keinen engen Zusammenhang mit den Grundnormen für die Funktionsweise des politischen Gemeinwesens aufweist « ${ }^{42}$

Im Bereich des Strafrechts liegen nicht nur eine Reihe von Beiträgen von Strafrechtlern zu Themen der Durchdringung des Strafrechts und des Strafprozessrechts durch das Verfassungsrecht vor, ${ }^{43}$ sondern auch ein rechtsvergleichendes Themenheft der Zeitschrift Revue des sciences criminelles, das auf eine Anregung ihrer Herausgeberin zurückgeht. ${ }^{44}$

Die gleiche Feststellung kann selbstverständlich auch in Bezug auf die übrigen Gebiete des öffentlichen Rechts getroffen werden, seien es das öffentliche Finanzwesen ${ }^{45}$, das Verwaltungsrecht ${ }^{46}$ oder die Grundrechte (libertés publiques) ${ }^{47}$. Es ist heutzutage normal geworden, den Bezug zum Verfassungsrecht herzustellen. In diesem Zusammenhang sei hier stellvertretend eine besonders bedeutsame (weil von einem Mitglied des Staatsrates stammende) Äußerung zitiert:

»Die Verfassungsnormen sind im Verwaltungsrecht allgegenwärtig, und der Übergang vom einen zum anderen ist in der Realität kaum mehr spürbar, seitdem und in dem Maße in dem sich durch die Rechtsprechung des Verfassungsrates die verfassungsrechtlichen Grundlagen des öffentlichen Rechts fortentwickelt haben.« 48

2. Es ist angezeigt, in wenigen Worten nachzuzeichnen, worin die verfassungsrechtliche Durchdringung eines Rechtsgebietes genau besteht.

Zunächst bedeutet dieses Phänomen, dass das betreffende Rechtsgebiet nunmehr verfassungsrechtliche Grundlagen besitzt, die es in jeder Lehrveranstaltung und bei jeder Forschungsarbeit zu berücksichtigen gilt, wenn der geltende Stand des Rechts nicht verkannt werden soll. Im Verwaltungsrecht zum Beispiel können Themen wie Körperschaften des öffentlichen Rechts, Verwaltungsstrafen, Mittlerorganisationen, das Legalitätsprinzip usw. nicht mehr ohne Bezugnahme auf die Verfassung und deren verfassungsgerichtliche Auslegung behandelt werden. Des Weiteren ist es im Strafrecht und im Strafprozessrecht unmöglich geworden, das Rückwirkungsverbot, die Unschuldsvermutung, die Rechte der Verteidigung usw. losgelöst von den verfassungsrechtlichen Bestimmungen und Grundsätzen zu analysieren, die »das materielle Strafrecht einschränken $\ll^{49}$ Diese Beispiele ließen sich beliebig fortsetzen, auch in Bezug auf andere Rechtsgebiete.

Die verfassungsrechtliche Durchdringung eines Rechtsgebietes bedeutet des Weiteren, dass das betreffende Gebiet unter dem Einfluss des Verfassungsrechts Veränderungen erfährt. So kann beispielsweise das bürgerliche Recht durch das verfassungsrechtliche Gleichheitsgebot, insbesondere durch den Grundsatz der Gleichheit zwischen Männern und Frauen, einen Wandel durchlaufen, wie dies z. B. in Deutschland geschehen ist. ${ }^{50}$ Auch das Gesellschaftsrecht oder das Recht des Öffentlichen Dienstes werden durch das Gleichheitsgebot Wandlungen erleben. Diese Wandlungen vollziehen sich dabei immer zugunsten einer größeren Freiheit des Einzelnen und einer stärkeren Berücksichtigung von dessen Grundrechten. 
60 Allerdings ist anzumerken, dass der Grad der Durchdringung durch das Verfassungsrecht von Rechtsgebiet zu Rechtsgebiet unterschiedlich ausfällt: in manchen ist diese Durchdringung tiefer als in anderen, wobei mit der Zeit eine gewisse Angleichung stattfindet bzw. stattfinden wird. Tatsächlich hängt diese Durchdringung auch von der Gesetzgebungstätigkeit in diesem oder jenem Rechtsgebiet ab: Verabschiedet der Gesetzgeber in einem bestimmten Rechtsgebiet besonders viele Gesetze, wird auch der Verfassungsrat häufiger die Gelegenheit erhalten, zu diesen Fragen $\mathrm{zu}$ entscheiden und in Bezug auf diesen Rechtsbereich die Verfassung auszulegen. Von diesem Standpunkt aus betrachtet, würde die 1989 vom Präsidenten des Verfassungsrates sowie vom Präsidenten der Republik vorgeschlagene Reform zweifelsohne dazu führen (so sie denn verabschiedet würde), diesen Prozess der verfassungsrechtlichen Durchdringung erheblich zu beschleunigen.

61 Deren Fortschritte lassen sich offensichtlich am besten durch eine Analyse der Rechtsprechung der Verwaltungsgerichte und der ordentlichen Gerichte, aber auch durch eine Beschäftigung mit der institutionellen Praxis und der Gesetzgebungspraxis verfolgen und messen.

62 Doch geht die seit über fünfzehn Jahren beobachtete Entwicklung noch darüber hinaus: tatsächlich ist inzwischen die Frage immer stärker berechtigt, ob die Unterscheidung zwischen öffentlichem Recht und Privatrecht noch begründet ist.

\section{B. Die zunehmende Relativierung der Unterscheidung zwischen öffentlichem Recht und Privatrecht}

63 1. Die meisten Rechtslehrbücher beginnen mit einer Darstellung der Rechtsgebiete, die auf einer fundamentalen Unterscheidung zwischen öffentlichem Recht und Privatrecht beruht, fahren anschließend mit einer Darstellung des ausgewählten Rechtsgebiets innerhalb des öffentlichen Rechts oder des Privatrechts fort und schließen mit einer Hervorhebung der Eigenständigkeit des behandelten Rechtsgebiets, das ganz selbstverständlich seine eigenen Grundsätze und Grundlagen besäße, die natürlich nicht dem Einfluss anderer Rechtsdisziplinen zu verdanken seien.

Eine derartige Darstellungsweise war noch vorstellbar, als die einzelnen Rechtsgebiete noch keinen gemeinsamen Unterbau bzw. keine gemeinsame Grundlage besaßen. Damals wurde die Verfassung in der Tat nicht als Gesamtheit von Normen aufgefasst, die als Grundlage der Rechtsordnung fungieren könnten, und allgemeine Rechtsgrundsätze wurden bald von der Rechtsprechung des Staatsrates, bald von derjenigen des Kassationsgerichtshofes herausgearbeitet; selbst wenn diese allgemeinen Rechtsgrundsätze sich manchmal überschnitten, waren sie doch keine gemeinsamen oder einheitlichen Grundsätze für das öffentliche Recht und für das Privatrecht (manchmal gar galten diese Grundsätze nicht einmal in einheitlicher Weise für alle Rechtsgebiete aus einer dieser beiden Rechtsordnungen ${ }^{51}$ ). Diese Zeit ist nun überwunden, denn die Normen der Verfassung gelten gleichermaßen für das Privatrecht und für das öffentliche Recht und bilden einen gemeinsamen Stamm, mit dem die einzelnen Rechtsgebiete verbunden sind, ohne dass es notwendig wäre, zwischen privatrechtlichen und öffentlich-rechtlichen Rechtszweigen $\mathrm{zu}$ unterscheiden.

Vor zehn Jahren konstatierten wir ${ }^{52}$ eine $» B e w e g u n g$ hin zu einer Wiedervereinigung des öffentlichen Rechts, die sich aus der Entwicklung des Verfassungsrechts hin zu 
einem Grundsockel« ergab. Heutzutage hat sich diese Bewegung nicht bloß stark weiterentwickelt, sondern greift auch auf das Privatrecht über. Dessen ist sich allerdings noch nicht jeder Jurist bewusst geworden, und es dürfte noch eine Weile dauern, bis die verfassungsrechtliche Denkweise bei allen, deren Aufgabe die Anwendung oder Auslegung des Rechts ist, zu einem normalen Reflex wird.

2. Worum es geht, ist die Einheit der Rechtsordnung und deren Identität beziehungsweise Einzigartigkeit.

Von dem Moment an, wo die allgemeinen Rechtsgrundsätze - zumindest diejenigen der obersten Ebene - mehr und mehr von Verfassungsnormen absorbiert und ersetzt worden sein werden, werden auch die Gefahren verschwinden, die sich daraus ergeben, dass mehrere Rechtsordnungen nebeneinander bestehen.

Das Recht wird dann nur noch eine einzige Verfassung haben und ein einziges Gebälk, um es zu stützen.

Durch eine derartige Entwicklung werden die einzelnen Rechtszweige nicht ihre Eigenheiten einbüßen, denn wie weiter oben bereits beobachtet, festigt und bestätigt die Verfassungsrechtsprechung oftmals Lösungsansätze, die bereits von der Rechtsprechung der Verwaltungsgerichte oder der ordentlichen Gerichte herausgearbeitet wurden.

Es kann sogar behauptet werden, dass diese Festigung geeignet ist, die Besonderheiten des nationalen Rechts trotz des Eindringens gemeinschaftsrechtlicher und völkerrechtlicher Normen in die innerstaatliche Rechtsordnung zu bewahren. Die verfassungsrechtliche Durchdringung aller Rechtsgebiete erlaubt es, diese vor einer gemeinschaftsrechtlichen Vereinheitlichung zu schützen.

Das in diesem Beitrag beschriebene moderne Verfassungsrecht ist nicht mehr bloß ein Verfassungsrecht der westlichen Demokratien, das als solches nicht auf die Staaten des Ostblocks oder des Südens übertragbar wäre.

Die Ereignisse und Umwälzungen, die sich in den Volksdemokratien vollziehen und auch vor bestimmten politischen Ordnungen in der Dritten Welt, so z. B. in Algerien, nicht haltmachen, zeigen, dass in allen Ländern eine ähnliche Entwicklung hin zu gleichen Auffassungen von Demokratie und Verfassungsstaat stattfindet. Die Forderung nach Pluralismus und der Errichtung eines Rechtsstaats ist nicht mehr, so scheint es, ausschließlich auf freiheitliche und kapitalistische Gesellschaftsordnungen beschränkt.

Überall dort, wo diese Forderung laut wird, wird auch die Einrichtung einer Verfassungsgerichtsbarkeit verlangt: nicht, weil es sie ein weiterer Baustein aus dem Verfassungsbaukasten wäre, sondern weil es sich dabei um eine unabdingbare Voraussetzung für die Verwirklichung und die Bewahrung des Rechtsstaats handelt.

4 In jedem seiner drei Aspekte (der Organe, des Normengefüges und der Grundrechte) scheint das Verfassungsrecht zum gemeinsamen Nenner aller Systeme zu werden, die für sich in Anspruch nehmen, demokratisch zu sein. Begrenzt auf den Aspekt der Staatsorganisation, konnte das Verfassungsrecht hingegen nur zersplittert und nationalspezifisch bleiben, selbst wenn es aus dem Blickwinkel der allgemeinen Staatstheorie betrachtet wurde, denn diese allgemeine Staatstheorie befasst sich in Frankreich im Wesentlichen mit den Institutionen des Staates.

Wenn die vorliegende Analyse bestätigt werden sollte - und sie wird es werden, wenn die französischen Verfassungsrechtler $\mathrm{zu}$ ihren ausländischen Kollegen aufschließen 
und nicht in ihrer Isolierung verharren wollen -, dann wird noch vieles Überkommene einer Revision unterzogen werden müssen.

\section{BIBLIOGRAPHIE}

Ackerman, B. (1994): »Dualistic Democracy«, in: Troper, M. / Jaume, L. (Hg.): 1789 et l'invention de la Constitution: Actes du colloque de Paris organisé par l'Association Française de Science Politique; 2, 3 et 4 mars 1989, Paris / Brüssel: LGDJ / Bruylant.

Atias, C. (1985): Epistémologie juridique, Paris: PUF.

Bacoyannis, C. (1989): Le principe constitutionnel de libre administration des collectivités territoriales, Dissertation, Aix-Marseille III.

Debbasch, R. (1988): Le principe révolutionnaire d'unité et d'indivisibilité de la République, Paris / AixMarseille: Economica / PUAM.

Delmas-Marty, M. (1989): »La jurisprudence du Conseil constitutionnel et les principes fondamentaux du droit pénal proclamés par la Déclaration de 1789«, in: La déclaration des droits de l'homme et du citoyen et la jurisprudence: colloque des 25 et 26 mai 1989 au Conseil constitutionnel, Paris: PUF.

Dworkin, R. (1984 [1977]): Bürgerrechte ernstgenommen, übersetzt von Ursula Wolf, Frankfurt a. M.: Suhrkamp.

Eisenmann, C. (1950-1951): Cours de Droit constitutionnel comparé, 1950-1951, Paris: Le Cours de droit.

Eisenmann, C. (1986 [1928]): La justice constitutionnelle et la Haute Cour constitutionnelle d'Autriche, 2. Aufl., Paris / Aix-Marseille: Economica / PUAM.

Escarras, J. C. (1986): »Sur deux études italiennes: de la communicabilité entre systèmes italien et français de justice constitutionnelle«, Annuaire international de justice constitutionnelle, Bd. II, S. 1533.

Favoreu, L. (1980): »L'apport du Conseil constitutionnel au droit public«, Pouvoirs, Nr. 13, S. 17-31.

Favoreu, L. (1982 [1978]): Le domaine de la loi et du règlement, 2. Aufl., Paris / Aix-Marseille: Economica / PUAM.

Favoreu, L. (1982): »L'influence de la jurisprudence du Conseil constitutionnel sur les diverses branches du droit «, in: Conac, G. / Maisl, H. / Vaudieux, J. (Hg.): Itinéraires: études en l'honneur de Léo Hamon, Paris: Economica, S. 235 ff.

Favoreu, L. (1988): „Dualité ou unité de l'ordre juridique«, in: Conseil constitutionnel et Conseil d'Etat: colloque des jeudi 21 et vendredi 22 janvier 1988 au Sénat, Salle Médicis, Palais du Luxembourg, Paris: LGDJ, S. $145 \mathrm{ff}$.

Favoreu, L. (1992): „Rapport introductif«, in: Maus, D. / Favoreu, L. / Parodi, J.-L. (Hg.) : L'écriture de la Constitution de 1958: actes du colloque du 30ªnniversaire, Aix-en-Provence, 8, 9, 10 septembre 1988, Paris / Aix-Marseille: Economica / PUAM. 
François, B. (1990): »Le juge, le droit et la politique: éléments d'une analyse politiste«, Revue française de droit constitutionnel, Nr. 1, S. 49-69.

Fromont, M. (1975): „Les droits fondamentaux dans l'ordre juridique de la l'Allemagne fédérale«, in: Waline, M. (Hg.): Recueil d'études en hommage à Charles Eisenmann, Paris: éd. Cujas.

Gaia, P. (1990): Le Conseil constitutionnel et le contrôle de l'intégration des engagements internationaux dans l'ordre juridique interne, Dissertation, Aix-Marseille III.

Garcia de Enterria, E. (1985): La Constituciòn como norma y el Tribunal constitucional, 3. Aufl., Madrid: Civitas.

Gaxie, D. (1989): Jeux croisés, Droit et politique dans la polémique sur le refus de signature des ordonnances par le président de la République, in: Lochak, D. et al. (Hg.): Les usages sociaux du droit, Paris: PUF, S. 209 ff.

Hamon, F. / Wiener, C.: »Justice - Justice constitutionelle«, in: Cours constitutionnelles, Encyclopaedia Universalis, abrufbar unter: https://www.universalis.fr/encyclopedie/coursconstitutionnelles/.

Lavau, G. (1989): »Profession politiste. Entretien avec Georges Lavau«, Interviewer F. Haegel und Jean Baptiste Legavre, Politix, Nr. 7-8, S. 132-138.

Le Calvez, J. (1985): »Les principes constitutionnels en droit pénal«, JCP, Nr. 3198.

Legeais, R. (1981): »La Constitution et le droit pénal dans le système juridique français«, Revue internationale de droit comparé (Sondernummer), Bd. III, S. 621-631; jetzt auch in: Mélanges R. Legeais, Paris: Éd. Cujas, S. 189-200.

Luchaire, F. (1987): La protection constitutionnelle des droits et des libertés, Paris: Economica.

Meny, Y. (1988): Politique comparée: les démocraties: Allemagne, États-Unis, France, Grande-Bretagne, Italie, 2. Aufl., Paris: Montchrestien.

Michaut, F. (1989): „L'inscription de la décision judiciaire dans un système juridique. Réflexions sur l'évolution de la doctrine américaine contemporaine«, Revue du droit public, Nr. 4, S. 10091059.

Miclo, F. (1982): Le régime législatif des DOM et l'unité de la République, Paris / Aix-Marseille: Economica / PUAM.

Murphy, W. F. (1989): „Constitutions, Constitutionalism, and Democracy«, Vortrag auf der Tagung des American Coucil of Learned Societies (ACLS), Berlin 1989; jetzt in: Constitutionalism \& Democracy: Transitions in the Contemporary World, Oxford: Oxford University Press, 1993.

Philip, L. (1984): »La constitutionnalisation du droit budgétaire français«, in: Beck, B. et al. (Hg.): Études de finances publiques. Mélanges en l'honneur de M. le Professeur Paul Marie Gaudemet, Paris: Economica, S. 49-62.

Pizzorusso, A. (1977): Delle Fonti del Diritto, in: Scialoja, A. / Branca, G. (Hg.): Commentario del Codice civile, Bologna / Rom: Zanichelli.

Prelot, M. / Boulouis J. (1987): Institutions politiques et droit constitutionnel, 10. Aufl., Paris: Dallos. Stirn, B. (1989): Les sources constitutionnelles du droit administratif, Paris: LGDJ.

Troper, M. (1974): »Le probleme de l'interprétation et la théorie de la supra-légalité constitutionnelle«, in: Recueil d'etudes en hommage à Charles Eisenmann, Paris: Éd. Cujas. 
Troper, M. (1990): »Justice constitutionnelle et démocratie«, Revue française de Droit constitutionnel, Nr. 1, S. 31-48.

Vedel, G. (1949): Droit constitutionel, Paris: Sirey.

Vedel, G. (1984a): „Le précédent judiciaire en droit public français«, in: RIDC, Sonderheft, VI, S. $283 \mathrm{ff}$.

Vedel, G. (1984b): Vorwort zu Beck, B. et al. (Hg.): Études de finances publiques. Mélanges en l'honneur de M. le Professeur Paul-Marie Gaudemet, Paris: Economica.

Vedel, G. (1990): »La continuité constitutionnelle en France de 1789 à 1989«, Revue française de droit constitutionnel Nr. 1, S. 5-15.

Zagrebelsky, G. (1986): »La doctrine du droit vivant«, Annuaire international de justice constitutionnelle, Bd. II, S. 54-77.

\section{NOTES}

1. Favoreu (1980), S. 17 f.

2. Fachtagung in St. Maur, Februar 1980.

3. S. unsere Bemerkungen in der Revue du Droit Public, 1989, Nr. 2, S. 407; $90 \%$ der Rechtsprechung des Verfassungsrates sind in den Jahren zwischen 1980 und 1989 ergangen.

4. Auf diese Veränderungen wurde bereits hingewiesen von Favoreu (1982), S. 235 f.

5. Siehe insbesondere die erstaunlichen Ausführungen von Gaxie (1989), S. 225, Fn. 8: »Man denke an dieser Stelle nur an die intellektuelle bzw., allgemeiner, an die akademische Strategie von Louis Favoreu. "Diese eher kryptische Andeutung wird in Fußnote 42 aufgeklärt: »Auch hier ist diese intellektuelle Strategie untrennbarer Bestandteil einer akademischen Strategie, die danach strebt, die Tradition der Rechtsschulen wiederaufleben $\mathrm{zu}$ lassen und innerhalb derselben die klassische Vorlesung zum ıVerfassungsrecht >Verfassungsprozessrecht $\iota$ zu ersetzen.«

6. In diesem Sinne, s. Garcia de Enterria (1985).

7. Dieses Phänomen haben wir bereits häufiger hervorgehoben, und dabei auch den Begriff »Verrechtlichung« verwendet, s. insbesondere Favoreu (1980).

8. Troper (1974), S. 133 und (1990), S. 38 ff.

9. Vedel (1984a).

10. Zur Vermengung von Recht und Rechtswissenschaft vgl. die Bemerkungen von Atias (1985), S. 31.

11. Auch wenn die wissenschaftliche Genauigkeit der Termini Anlass zu Diskussionen geben mag. 12. Inhaltlich handelt es sich dabei im Wesentlichen um eine allgemeine theoretische Behandlung politischer Institutionen aus französischer Sicht.

13. Selbst Charles Eisenmann vertritt diese Ansicht noch 1950: Er wendet sich gegen die Sichtweise Prélots, wonach »das Verfassungsrecht die Struktur der Organe und des Staatsapparates als Ganzes zum Gegenstand habe, d. h. sowohl die Organisation der Verwaltung und des Gerichtswesens als auch der höheren Staatsorgane Parlament und Regierung «. Für Eisenmann hingegen gilt: »Es ist als überlieferte und berechtigte Tatsache festzuhalten, dass das >Verfassungsrecht sich ausschließlich mit Fragen und Bestimmungen zu den obersten Organen des Staates befasst, d. h. mit den Grundlagen der politischen Organisation des Staates« (Vorlesung zum Vergleichenden Verfassungsrecht; Eisenmann [1950-1951], S. 37).

14. Entscheidungen Nr. 82-137 DC und Nr. 82-138 DC, Dezentralisierung, in: GD, 5. Aufl., Nr. 34. 
15. Entscheidung Nr. 80-119 DC, Gültigerklärung von Verwaltungsverordnungen, in: GD, 5. Aufl. Nr. 31.

16. Entscheidung Nr. 86-224 DC, Wettbewerbsbehörde, in: GD, 5. Aufl., Nr. 43.

17. In den französischen Rechtswissenschaften wird die Frage der Rechtsquellen meist im Rahmen der Einführung in die Rechtswissenschaft thematisiert, die im Übrigen oftmals eher eine Einführung in das Zivilrecht darstellt.

18. Es genügt, hier auf den Stellenwert hinzuweisen, der den Rechtsquellen im deutschen oder im italienischen Verfassungsrecht eingeräumt wird. So kann beispielsweise festgestellt werden, dass im Kommentar zum italienischen Zivilgesetzbuch der Abschnitt über die »Rechtsquellen« aus der Feder des Verfassungsrechtlers Alessandro Pizzorusso stammt, der diesem Thema ungefähr sechshundert Seiten widmet; Pizzorusso (1977).

19. S. unseren einführenden Bericht zu L'écriture de la Constitution, dem Symposium über die Abfassung der Verfassung, Aix-en-Provence, September 1988; Favoreu (1992).

20. Dieses Forschungsgebiet haben wir seit 1977 und dem Symposium über »Das Gesetz und die Rechtsverordnung " (Le domaine de la loi et du règlement, 1978, Neuaufl. 1982) zu erschließen versucht und auch andere dazu ermuntert, sich ihm $\mathrm{zu}$ widmen, so durch Dissertationen. Genannt seien beispielsweise zu Artikel 37, Absatz 2 (Fertigstellung steht bevor), Artikel 38 (in Vorbereitung), Artikel 41 [É. Oliva, "L'article 41 de la Constitution du 4 octobre 1958: initiative législative et Constitution«, 1997], Artikel 46 [J.-C.Car, "Les lois organiques de l'art. 46 de la Constitution du 4 octobre 1958«, 1999], Artikel 53 und 54 (P. Gaia, Le Conseil constitutionnel et le contrôle de l'intégration des engagements internationaux dans l'ordre juridique interne, Diss. AixMarseille III, 1990), Artikel 72 (C. Bacoyannis, Le principe constitutionnel de libre administration des collectivités territoriales, 1993), Artikel 73 (F. Miclo, Le régime législatif des DOM et l'unité de la République, 1982), Artikel 74 (in Vorbereitung), oder auch Artikel 92 (in Vorbereitung). Zu der Erforschung des Themas »Rechtsquellen«, insbesondere Artikel 2 der Verfassung, kann auch die Dissertation von R. Debbasch, Le principe révolutionnaire d'unité et d'indivisibilité de la République, Aix-Marseille III, 1987 (erschienen 1988) gezählt werden.

21. Siehe insbesondere unsere Rechtsprechungschronik in der Revue du droit public.

22. Eisenmann (1928), S. 24 f.

23. Dies ist bei allen neueren Verfassungen der Fall.

24. M. Prélot und J. Boulouis, Institutions politiques et droit constitutionnel, 10. Aufl., S. 33. Dies war die Ansicht Marcel Prélots. Jean Boulouis hingegen legte dar, wie sich die Dinge seitdem geändert haben. Er räumt ein, dass neben den Normen und den Grundfreiheiten nun eigentlich auch die Organisation der Verwaltung und das Gerichtswesen in Rahmen der Verfassungsrechtslehre thematisiert werden müssten, und fügt hinzu, dass »wenn man an (der) klassischen Definition festhält, dann nur aus einem Sachzwang zur Einteilung des Lehrstoffs, die allerdings immer weniger dem Stand des positiven Rechts und den inhaltlichen pädagogischen Anforderungen entspricht« (§ 21, Hervorhebung des Verfassers).

25. Diese Prüfung ist heute strenger als früher, insoweit heute beispielsweise auch geprüft wird, ob der Anforderung einer gerechten Einteilung der Wahlkreise genügt wurde; aber all diese Fragen fallen unter das die Institutionen gestaltende Verfassungsrecht.

26. Murphy (1989), S. 21. W. F. Murphy ist einer der bedeutendsten Experten in Sachen »judicial politics« in den Vereinigten Staaten.

27. Ackerman (1989).

28. Vedel (1990), S. 7.

29. Gaxie (1989).

30. Hierbei handelt es sich vornehmlich um die Rechtsprechungstätigkeit der Verfassungsgerichte in Deutschland, Österreich, Italien, Spanien, Portugal und Belgien, die bislang von der französischen Rechtslehre weitgehend unbeachtet geblieben sind und die wir seit 1985 vermehrt in der Zeitschrift Annuaire international de justice constitutionnelle (AIJC) $\mathrm{zu}$ 
behandeln suchen. Darüber hinaus sei auch die sehr umfangreiche rechtswissenschaftliche Literatur zu dieser Rechtsprechung in Westdeutschland, Italien und Österreich, sowie nunmehr auch in Spanien und Portugal erwähnt, die von den Politikwissenschaftlern wie im Übrigen auch von sich ausschließlich mit der Staatorganisation befassenden Juristen und den Rechtstheoretikern und Rechtsphilosophen vollständig unbeachtet gelassen wird.

31. Im Gegensatz zur Vorgehensweise der amerikanischen Politikwissenschaftler.

32. In diesem Sinne, s. François (1990), S. 52. Vgl. ebenfalls die klugen Betrachtungen von Georges Lavau (1989), S. 135: „Versuchen wir doch, diese etwas engen Diskussionen zu überwinden, die so oft von Missverständnissen und Vorurteilen verdüstert werden. Ich möchte darlegen, auf welche Weise so manches Mal terminologische Meinungsverschiedenheiten, Unzulänglichkeiten vorheriger Definitionen und theoretische Unterscheidungen sich überlagern.« Diese Worte, geschrieben in Bezug auf einen Streit unter Politikwissenschaftlern, sind ebenso zutreffend in Bezug auf einen Diskurs zwischen Politikwissenschaftlern und Juristen.

33. Aus diesem Grund halten wir die von Y. Mény ([1988], S. 417) sowie von F. Hamon und C. Wiener (Encyclopaedia Universalis, $V^{\circ}$ Verfassungsgerichte) vorgenommene Vermengung der Begriffe für nicht vereinbar mit der in der Rechtsvergleichung allgemein anerkannten Betrachtungsweise.

34. Für eine vertiefende Analyse dieser Betrachtungsweise vgl. Escarras (1986).

35. Zagrebelsky (1986), S. 54 f.

36. Escarras (1986).

37. Als jüngste Beispiele seien hier die Rechtsprechung zum Streikrecht im Öffentlichen Dienst oder auch die Rechtsprechung über verwaltungsgerichtlich verfolgbare Übertretungen wegen Beschädigung oder Störung der Verkehrswege genannt.

38. Einen hervorragenden Überblick liefert Michaut (1989), S. $1009 \mathrm{f}$.

39. Troper (1990), S. 31.

40. Favoreu (1980), S. 17 f.; Favoreu (1982), S. 235 f.

41. Diese Ansicht wird vom Autor dieses Beitrags nicht geteilt.

42. Guyon (1986), S. 606.

43. S. beispielsweise Legeais (1981), S. 621-631; Le Calvez (1985).

44. Revue des sciences criminelles, 1985, Nr. 4, S. 711-754.

45. Philip (1984), S. 49; vgl. auch die Einführung von Georges Vedel zu diesem Werk.

46. S. diesbezüglich die Anmerkungen von Georges Vedel in der Ausgabe dieser Zeitschrift (Vedel [1990]), S. 14.

47. Luchaire (1987).

48. Stirn (1989), S. 8.

49. So Delmas-Marty (1989), S. 154.

50. Fromont (1975), S. 61 f. M. Fromont zeigt dort in allgemeinerer Weise auf, inwieweit eine solche Veränderung sich in den unterschiedlichen Rechtsgebieten vollzieht.

51. Für eine eingehendere Analyse dieser Frage vgl. Favoreu (1988), S. 145 f.

52. Favoreu (1980), S. 23. 
INDEX

Mots-clés : droit constitutionnel, droit de la Constitution, constitution du droit

Schlüsselwörter : Verfassungsrecht, Recht der Verfassung, Verfassung des Rechts

\section{AUTEURS}

LOUIS FAVOREU

Louis Favoreu war Professor für Verfassungs- und Staatsrecht an der Universität Aix-enProvence / Marseille. Nähere Informationen finden Sie hier. 